\title{
Axial mechanical experiments of unbonded flexible pipes
}

\author{
Jun-Peng Liu ${ }^{1} \cdot$ Murilo Augusto Vaz ${ }^{2} \cdot$ Rong-Qi Chen $^{3} \cdot$ Meng-Lan Duan $^{1} \cdot$ Irving Hernandez $^{2}$
}

Received: 26 March 2020 / Published online: 9 September 2020

(c) The Author(s) 2020

\begin{abstract}
Axial structural damping behavior induced by internal friction and viscoelastic properties of polymeric layers may have an inevitable influence on the global analysis of flexible pipes. In order to characterize this phenomenon and axial mechanical responses, a full-scale axial tensile experiment on a complex flexible pipe is conducted at room temperature, in which oscillation forces at different frequencies are applied on the sample. The parameters to be identified are axial strains which are measured by three kinds of instrumentations: linear variable differential transformer, strain gauge and camera united particle-tracking technology. The corresponding plots of axial force versus axial elongation exhibit obvious nonlinear hysteretic relationship. Consequently, the loss factor related to the axial structural damping behavior is found, which increases as the oscillation loading frequency grows. The axial strains from the three measurement systems in the mechanical experiment indicate good agreement, as well as the values of the equivalent axial stiffness. The damping generated by polymeric layers is relatively smaller than that caused by friction forces. Therefore, it can be concluded that friction forces maybe dominate the axial structural damping, especially on the conditions of high frequency.
\end{abstract}

Keywords Flexible pipes $\cdot$ Hysteresis behavior $\cdot$ Structural damping $\cdot$ Mechanical experiment

\section{Introduction}

Unbonded flexible pipes are widely used in offshore oil and gas fields development, which consist of several functional layers made by different materials, as shown in Fig. 1. The fact that axial structural damping generated by friction between adjacent layers and within specific layers, viscoelastic properties of polymeric layers on condition of vessel stochastic oscillator is usually disregarded in global analysis may cause conservative results in deep-water development. Accordingly, waste of resources leads to rising costs (Silveira et al. 2011). Therefore, identification and quantification of the axial structural damping are necessary and important,

\section{Edited by Xiu-Qiu Peng}

Jun-Peng Liu

liujp@cup.edu.cn

1 College of Safety and Ocean Engineering, China University of Petroleum, Beijing 102249, China

2 Ocean Engineering Program, Federal University of Rio de Janeiro, Rio de Janeiro, Brazil

3 Engineering and Construction Department, China National Offshore Oil Corporation, Beijing 100010, China which can be characterized by the loss factor $\eta$ defined as ratio of dissipated energy loss per radian $W_{\mathrm{d}} / 2 \pi$ divided by the peak potential or strain energy $U$ (Ungar and Kerwin 1962). The expression of loss factor can be written as:

$\eta=\frac{W_{\mathrm{d}}}{2 \pi U}$

Figure 2 shows a general result for the mechanical behavior of a flexible pipe, in which the enclosed area denotes the dissipated energy in one cycle, while the hatched triangle area is the peak potential energy. Hence, capturing the axial mechanical responses of flexible pipe under axisymmetric loads seems to be an effective method to pursue the loss factor. However, quite a few nonlinear properties caused by geometric, material, contact conditions make it arduous. Both numerical and analytical studies have to make lots of assumptions and put forward a slice of interesting results in the past 40 years (Feret and Bournazel 1987; Witz and Tan 1992; Kebadze 2000; Custódio and Vaz 2002; Pesce et al. 2010; Sævik 2011; Ramos et al. 2014; Ramos and Kawano 2015; Ebrahimi et al. 2018). Although some advances like viscoelastic considered in model (Guedes 2010; Liu and Vaz 2016a; Santos et al. 2017, Liang et al. 2018, 2019), there is 

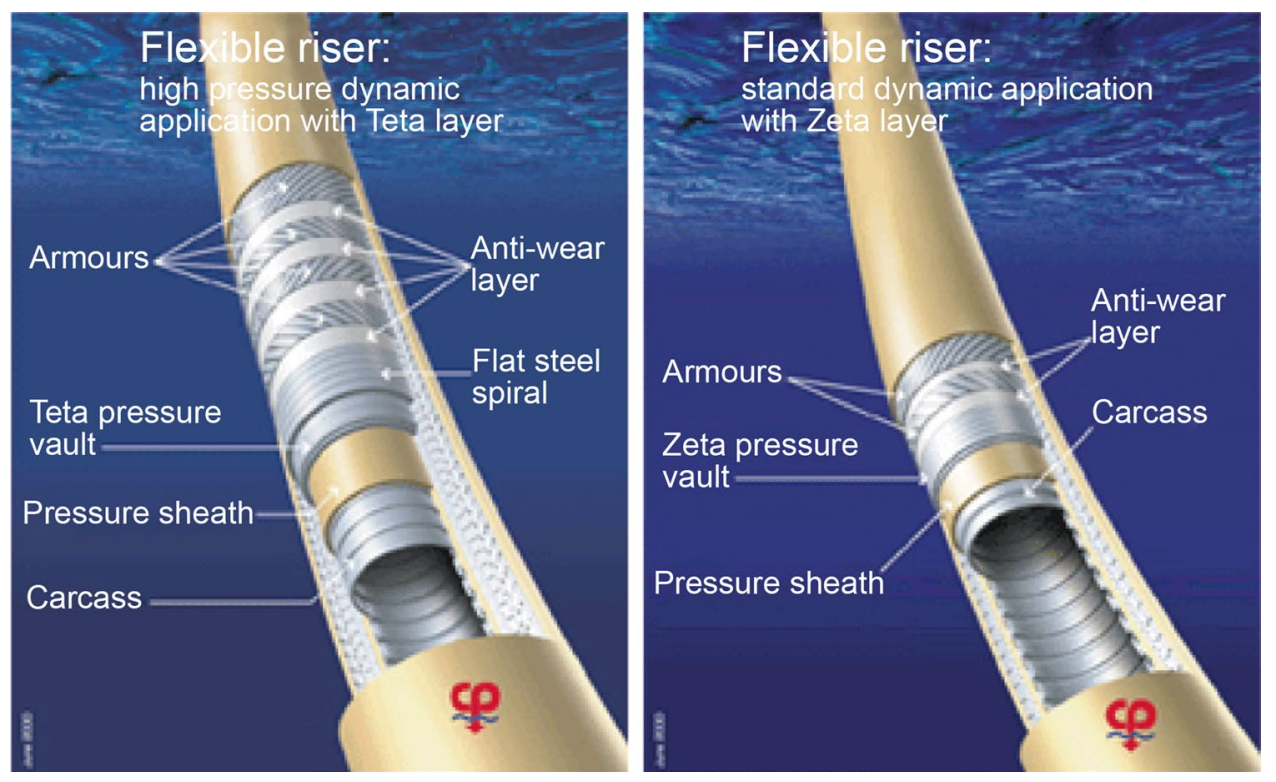

Fig. 1 Unbonded flexible pipe structure (TECHNIP)
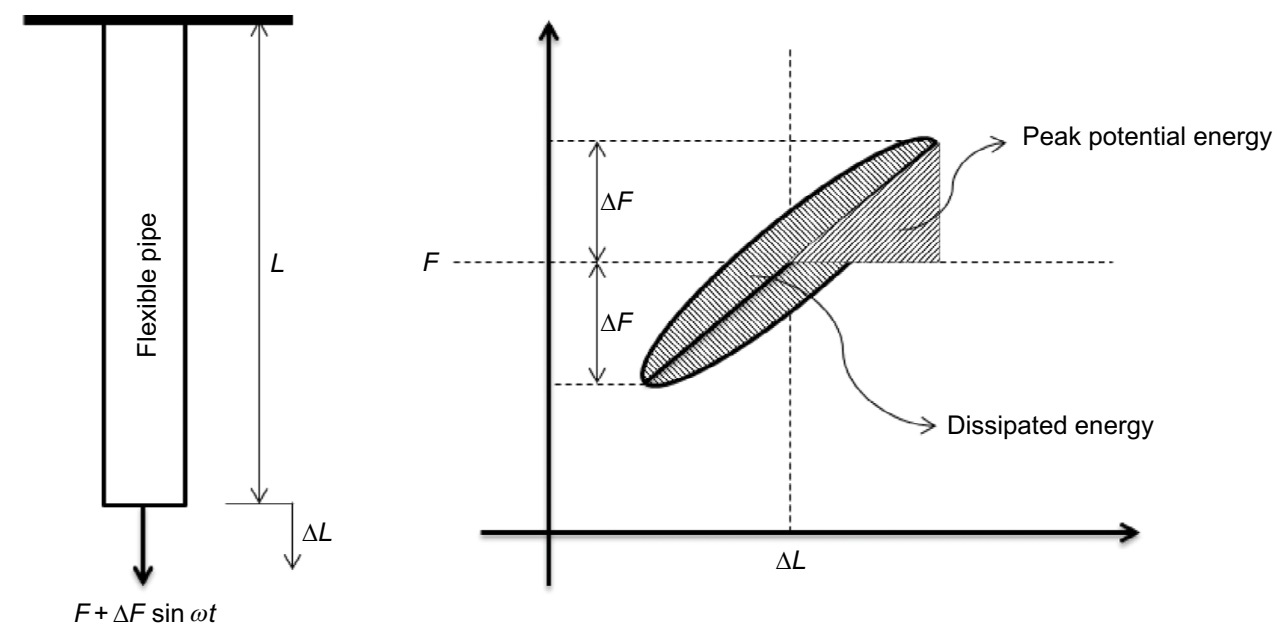

Fig. 2 Schematic of axial structural damping

still no robust theory which can accurately predict mechanical responses.

Mechanical experiment may be an effective method for obtaining the value of axial structural damping which can be considered as a combination of frictional and viscoelastic dissipation mechanisms. Liu and Vaz (2016b) presented a theoretical model for calculating viscoelastic damping; however, this model assumed no friction in the adjacent layers. So, the frictional damping may be evaluated by the difference between experiment results and viscoelastic model results. It can be imaged that if the loading velocities in test are very small, perhaps damping will basically come from friction forces; meanwhile, as we increase the speed (frequency), viscous damping grows.
Generally, industrial experiments employed in flexible pipes design are extremely time consuming and expensive, such as fatigue test or experiments for determination of collapse pressure. Therefore, it is necessary to ensure the experimental results to be reasonably deterministic. Hence, there are two purposes in this paper. The first is to design and conduct axial tension experiments to investigate whether axial structural damping behavior occurs and to evaluate the values of loss factor, where axial sinusoidal forces with different frequencies are applied on a 16-in flexible pipe. The second is to see how friction forces in flexible pipes would affect its axial structural damping behavior. Results provide nonlinear force-elongation relations, the slope of which, referred as to equivalent axial stiffness, characterizes the 
mechanical behavior of flexible pipes. Viscoelastic and frictional contributions to the axial structural damping can be assessed. It is seen that loss factor given by the experiment is much larger than the one from viscoelastic model, which means frictions within the interlocked layers and between the adjacent layers dominate the axial structure damping.

\section{Experimental setup}

This section introduces full-scale axial tensile mechanical experiments conducted at the Ocean Structure's Laboratory (NEO) in COPPE/UFRJ, aimed at verifying whether hysteresis occurs for flexible pipe subjected to oscillation loads, as well as the value of loss factor. There are three advanced horizontal test rigs in NEO, which can be utilized for tensile, compression, bending and torsional experiments, and its hydraulic driven actuators can provide oscillation forces at different frequencies. A schematic (plan view) drawing of the test rig employed in this test is shown in Fig. 3. The leftmost strain gauge is $2.5 \mathrm{~m}$ away from the front end fitting.

Specifically, this test rig was designed as a flexible hyperbaric chamber to perform cyclic bending tests in 6" ID flexible pipe samples at maximum pressure of 5000 psi. Figure 4a shows two axial force cylinders located in the forefront at top and bottom of the rig.

A 16-in flexible pipe assembled with two end fittings is installed in the horizontal test rig, as shown in Fig. 4b. The 9-m-length sample has a complex structure, including carcass, pressure armor and two tensile armors and several thermoplastic polymeric layers whose geometric and material parameters are shown in Table 1.

Upper and lower hydraulic cylinders shown in Fig. 4a can, respectively, provide a maximum tensile load of 500 $\mathrm{kN}$. In addition, to avoid gravitational effects resulting outof-plane curvatures, a supporting structure assembled with rollers is placed at middle position of frame (Fig. 4c). On the authority of the test rig compositions, the chosen test

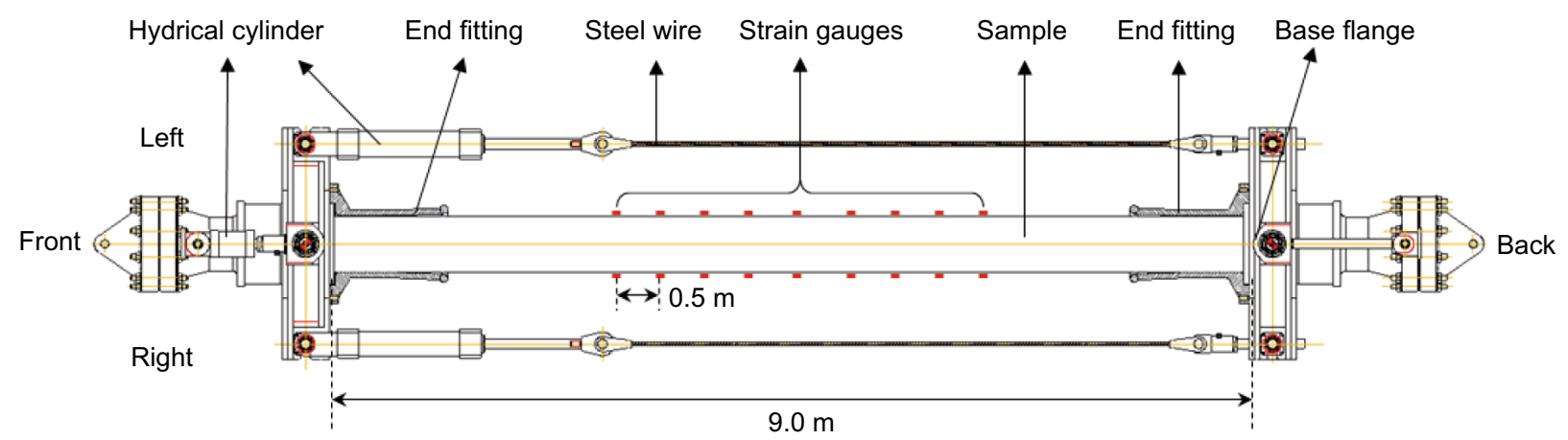

Fig. 3 Schematic plan view of the test rig
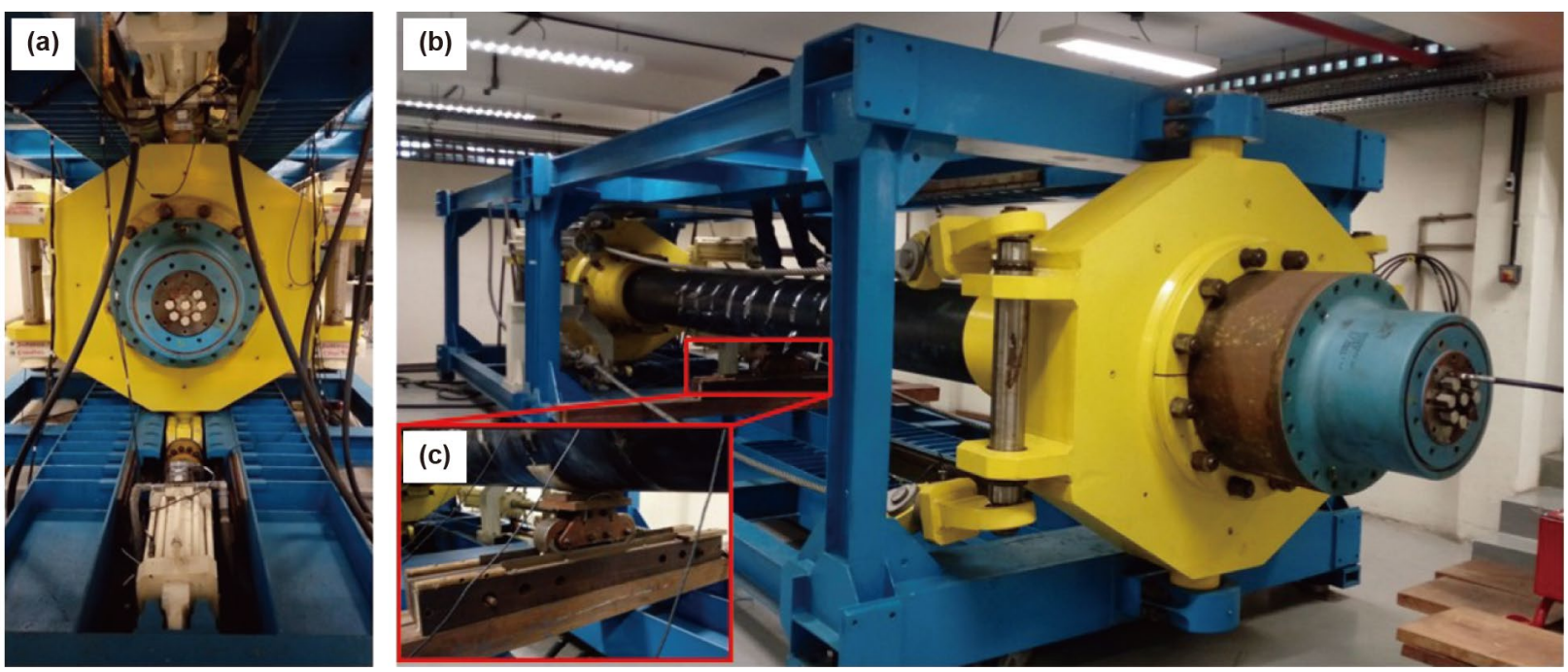

Fig. 4 Experimental setup (a, axial tensile hydraulic cylinder; $\mathbf{b}$, a full-scaled flexible pipe installed on test rig; $\mathbf{c}$, roller supporting structure) 
Table 1 Geometric parameters and material of the sample

\begin{tabular}{lllll}
\hline No. & Layer & Material & I.D, mm & O.D, mm \\
\hline 1 & Carcass & Stainless 316L & 406.40 & 427.10 \\
2 & Wear & PVDF & 427.10 & 439.10 \\
3 & Barrier & PVDF & 439.10 & 457.10 \\
4 & Pressure armor & Carbon steel & 457.10 & 481.08 \\
5 & Tape & PA11 & 481.08 & 484.12 \\
6 & Tensile armor & Carbon steel & 484.12 & 492.09 \\
7 & Tape & PA11 & 492.09 & 495.13 \\
8 & Tensile armor & Carbon steel & 495.13 & 503.11 \\
9 & Tape & Polypropylene & 503.11 & 503.70 \\
10 & Tape & High strength & 503.70 & 506.95 \\
& & glass filament & & \\
11 & Tape & Polypropylene & 506.95 & 507.54 \\
12 & Tape & Fabric & 507.54 & 508.36 \\
13 & Outer sheath & PA12 & 508.36 & 538.36 \\
\hline
\end{tabular}

principle is that the back end of sample can bend in horizontal plane but cannot move axially; however, the front side can be freely moved.

\section{Test scheme}

The tests are performed in unpressurized sample at room temperature in accordance with the following scheme.

Initially, the test sample is tensioned by an axial load of $500 \mathrm{kN}$ (each hydraulic cylinder provides $250 \mathrm{kN}$ ), gradually increased, to ensure no out-of-plane curvature caused by gravitational effect on the sample, although the roller supporting structure can apparently reduce the effect at middle position.

Secondly, given the creep behavior, once the value of tension force reaches $500 \mathrm{kN}, 20$ cycles of sinusoidal tension force $\Delta F$ with amplitude $50 \mathrm{kN}$ and variable frequency $\omega$, i.e., $\Delta F=50 * \sin (\omega t) \mathrm{kN}$, are immediately applied on the sample. Such process can be accurately controlled by the MTS system. Simultaneously, the data acquisition measurement equipment starts running.

Finally, the force is released and the sample returns to its original status. An interval between tests of at least $10 \mathrm{~min}$ is ensured to avoid residual strains and to restore the environmental temperature in the sample.

In order to guarantee the validity of the experimental data, the test is repeated twice and the average values of those three sets of the data are taken as the final representative results. The tests are performed for exciting frequencies $2 \pi / 7,2 \pi / 12,2 \pi / 17,2 \pi / 22 \mathrm{rad} / \mathrm{s}$ to check the influence of frequency on the mechanical behavior of the sample.

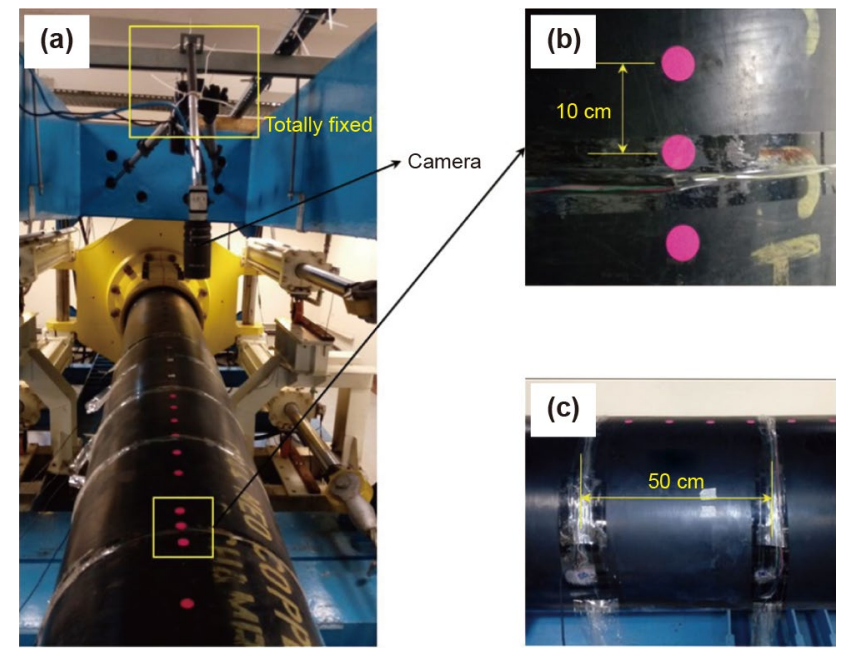

Fig. 5 Camera and strain gauges

\section{Measurement and instrumentation}

The key parameters to be measured in this experiment are the axial force and axial strain. Nine pairs of strain gauges are posted on the external surface of sample, symmetrically located in the left and right sides, as shown in Fig. 3. The interval between the near pair is $50 \mathrm{~cm}$, as shown in Fig. $5 \mathrm{c}$.

Moreover, other two kinds of instrumentations are used: (a) linear variable differential transformer-LVDT, which is coupled to the hydraulic drive system for displacement measurement, and/or (b) optical devices for particle tracking, which is uncommonly used in mechanical experiments of flexible pipes. Accordingly, it is necessary to briefly introduce this method.

\subsection{Particle-tracking gauge principle}

The principle of the particle-tracking system is that specific colored particles are tracked using an HSB color scheme segmentation to provide a buffer of binary images (Ye et al. 2013; Kumar et al. 2012; Pérez et al. 2002), and then the image particle tracking (IPT) is performed, as shown in Fig. 6.

As an image analysis challenge, the purpose of IPT is to segment and follow over time characteristic structures. Each particle is segmented over the entire buffer of acquired frames, and its trajectory is measured by assigning it an identity over these frames. Results such as velocity, total displacement and diffusion characteristics are then either visualized or processed to yield further analysis. The particle-tracking algorithm is written in JAVA language based on the Particle Analysis plug-in of ImageJ software (Schneider et al. 2012), because it can deal with multiple particle analysis over stack of images. The binary images, conformed by dark objects over 

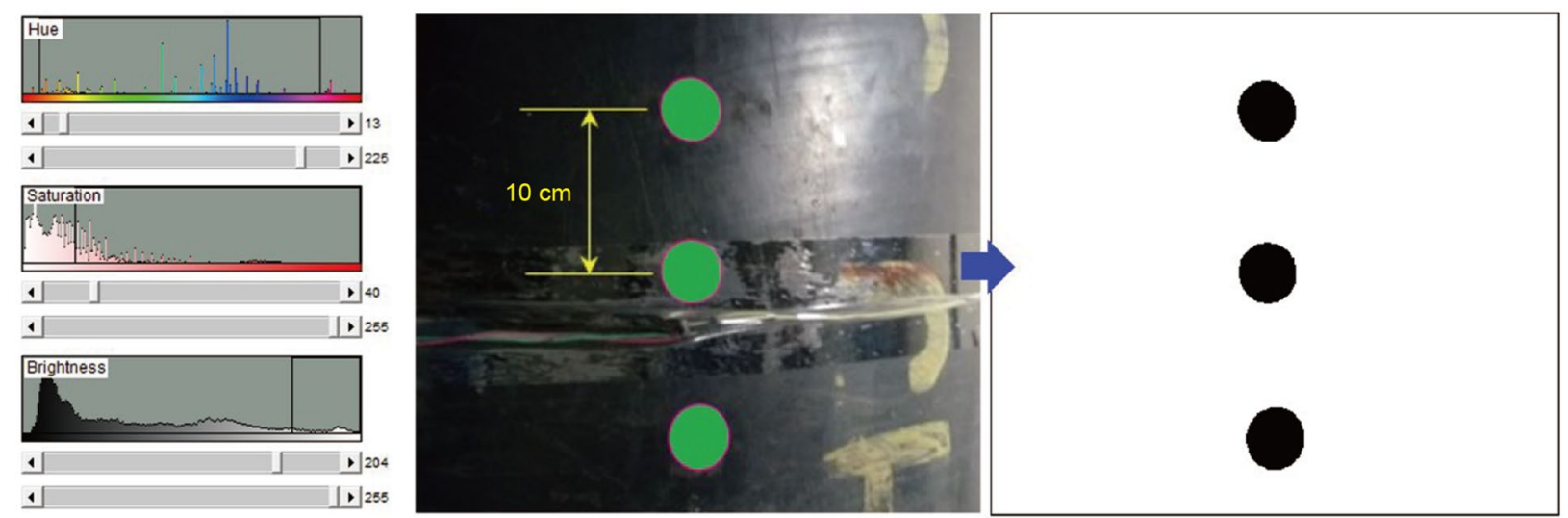

Fig. 6 Thresholding process for target tracking by HSB color scheme segmentation

a bright background, for which the object contour shape is not important, concern on extracting information from the $(X$, $Y)$ coordinates over time of the detected particles. Chenouard et al. (2014) carried out a comparative study of many particle tracking tools and provided the mathematical algorithms of particle tracking.

\subsection{Particle-tracking gauge setup}

The particle-tracking gauge monitoring system consists of three particles with a characteristic color and spaced 100 [mm]. The particles were positioned at the top center of the flexible pipe and the second one is at mid-length. The strain was measured in all possible combinations between particles, and finally it was considered the average of the inter-particle strains as final strain in the mid-length section.

\subsection{Hardware}

The hardware system comprises of three photoluminescent markers, a CMOS 5[MPX] professional camera (Fig. 5a) with external triggering synchronizer and a Corel i5 notebook running Windows OS.

\subsection{Configurations}

The configuration of acquisition considers 5 [fps] as frequency of image acquisition and $\{H \notin[13,225], S \in[40,255], B \in[204,255]\}$ segmentation color scheme for particle filtering, exporting the desired results in $\mathrm{a} *$.txt archive.

\section{Results and discussions}

The axial global strains are obtained dividing the displacements from the LVDT by the sample length. For each loading frequency, three groups of experiments are conducted, and mean values are shown in Fig. 7a for the axial strain varying with time. Clearly, the axial strains exhibit a rising trend, especially at the early stages, implying that creep behavior indeed exists. This behavior leads to the observation of axial force-elongation nonlinear relationship, as shown in Fig. 7b. Along with the increase in loading time, the curve will gradually become stable. Eventually, ellipse-shaped curves presented by the red solid line in Fig. $7 \mathrm{~b}$ appear, the area of which determines the dissipated energy per cycle. Another interesting phenomenon can be found, the axial strain increases with a decrease of loading frequency probably due to material creep.

Results of axial strains given by strain gauges are reported in Fig. 8 for five values of loading frequency. Because the time in each experiment is not synchronized, it is convenient to carry out the normalization processing for the time axis. The axial strain is larger for frequency $\omega=2 \pi / 22 \mathrm{rad} / \mathrm{s}$. Moreover, intuitively, although the values of axial strain are a little smaller than LVDT, the amplitudes for each case are almost the same.

Particle-tracking technology may be influenced by many factors, such as noise, light and frame vibration. Therefore, peaks and valleys of axial strains exhibit instability, as shown in Fig. 9. The axial strain of the middle zone can also be measured by strain gauge 03 , which is 

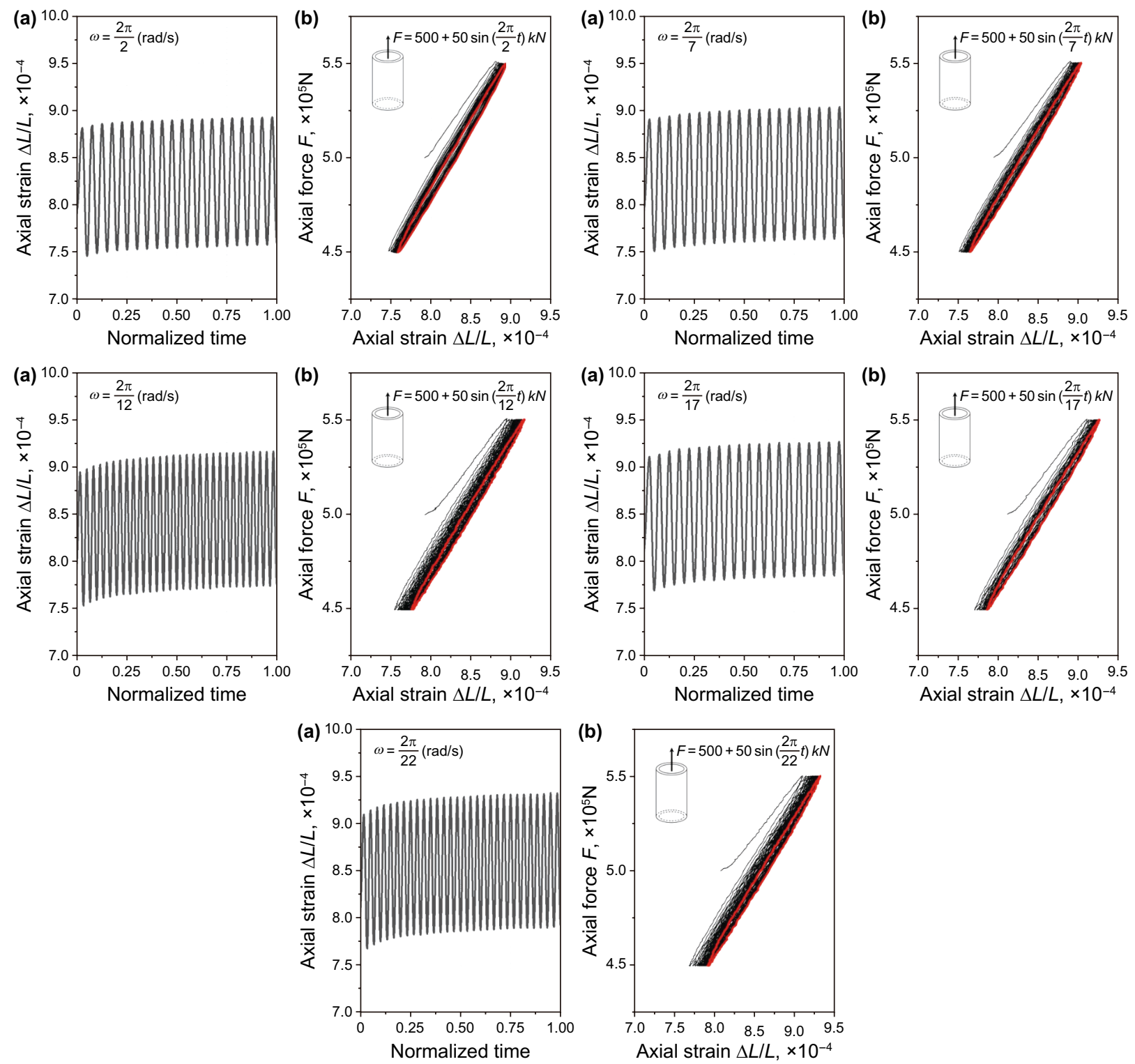

Fig. 7 Test results measured by LVDT on conditions of five different loading frequencies (a axial strains; b axial force-strain relations)

almost consistent with the particle-tracking one. However, both results are slightly smaller than the other strain gauges. Potentially, this could be interpreted as the friction force between the sample and support structure leading to enhance the resistance of elongation. Hence those results are not used as the global axial strain compared with theoretical model. Anyhow, the good agreement suggests that the results observed by strain gauges are reliable. Likewise, it can be imagined that although this technology does not have the ability to make accurate measurements, it is very helpful for the qualitative analysis of the experiment due to its low-cost and easy-operation. In addition, it is very useful for some experiments like bending test in which strain gauges cannot obtain results easily.
The results in frequency domain are equivalent to the ones of viscoelastic model in time domain for special case of time tending to infinity. Likewise, only in frequency domain can the loss factor be accurately calculated. Therefore, substituting the material parameters into the viscoelastic damping model given by Liu and Vaz (2016b) in frequency domain leads to the results for axial force-strain relation, which are as shown by dotted lines in Fig. 10. The results given by LVDT in the 20th cycle are represented by the solid lines in the same figure. Experimental average axial strain is larger than predicted by theoretical model, whereas the axial strain amplitudes are almost the same. Besides the loss factor, another parameter related to viscoelastic behavior is the equivalent axial stiffness, which can be defined as the 

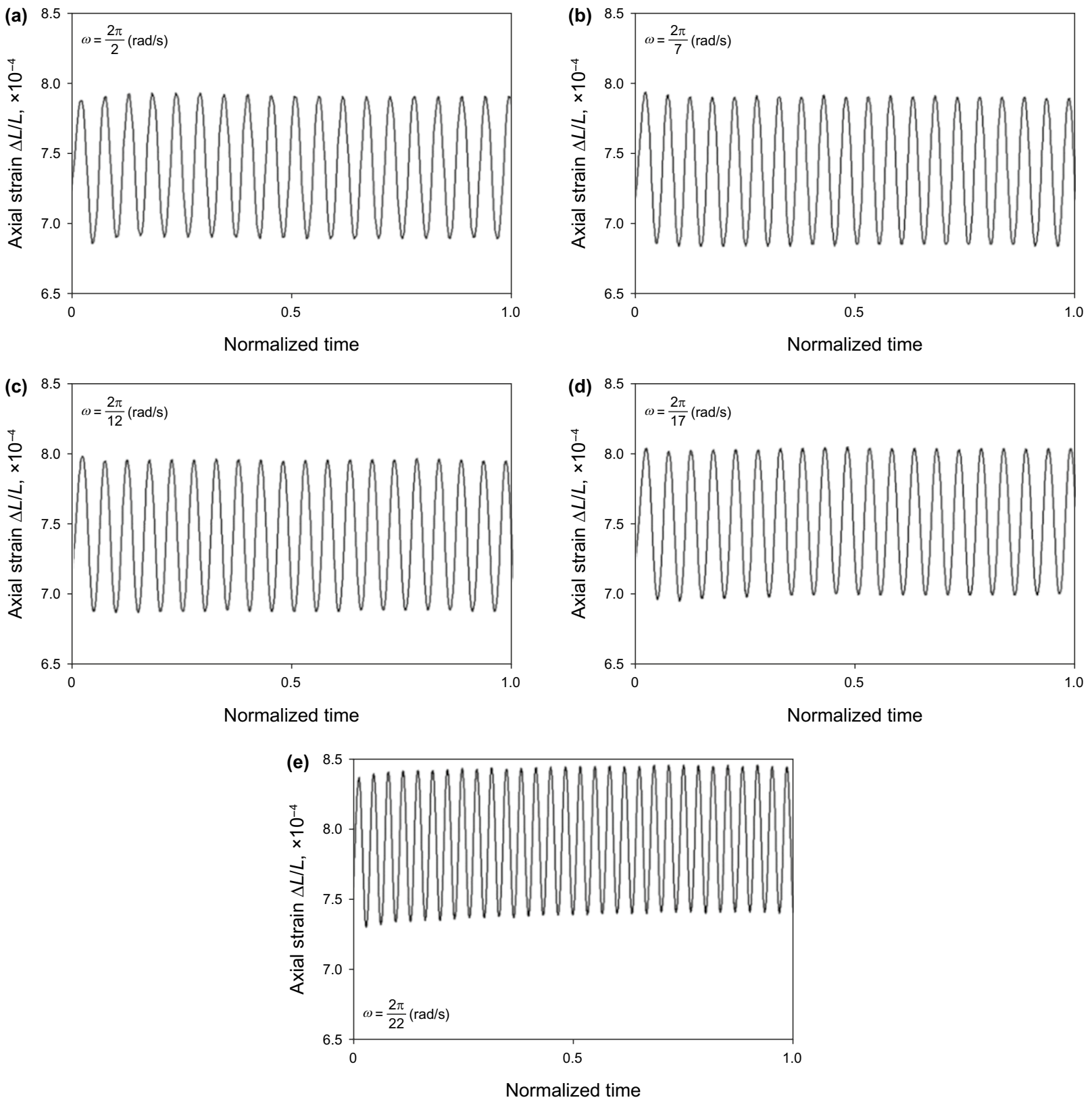

Fig. 8 Axial strains measured by one strain gauge for five different loading frequencies

axial force amplitude divided by the axial strain amplitude. Some variables associated with both parameters are shown in Table 2. It should be pointed out that difference of the loss factor captured by experiment and viscoelastic damping model are the damping generated by the frictional effects between the adjacent layers and self-locked layers such as carcass and pressure armor. The results for five loading frequencies are, respectively, 7.4\%, 5.8\%, 5.5\%, 5.6\%, 5.6\%.

Figure 11 shows comparisons of loss factor and equivalent axial stiffness. For each value of the loading frequency, the loss factor which can be accurately calculated by the model is apparently smaller than it caused by frictional effects, indicating that much more energy is lost because of friction given the fact that there is no sufficient difference for the peak potential energy between the experiment and model. That is to say, the friction dominates the axial structural damping behavior of flexible pipe. In addition, maximum value of loss factor provided by model maybe appears at some frequency between $2 \pi / 12$ and $2 \pi / 7$, but the experimental loss factor increases with a rise in frequency. Furthermore, the model results show that the equivalent axial stiffness which grows with an increase in frequency is larger than the experiment. However, the insignificant difference suggests that the viscoelastic model for calculating 

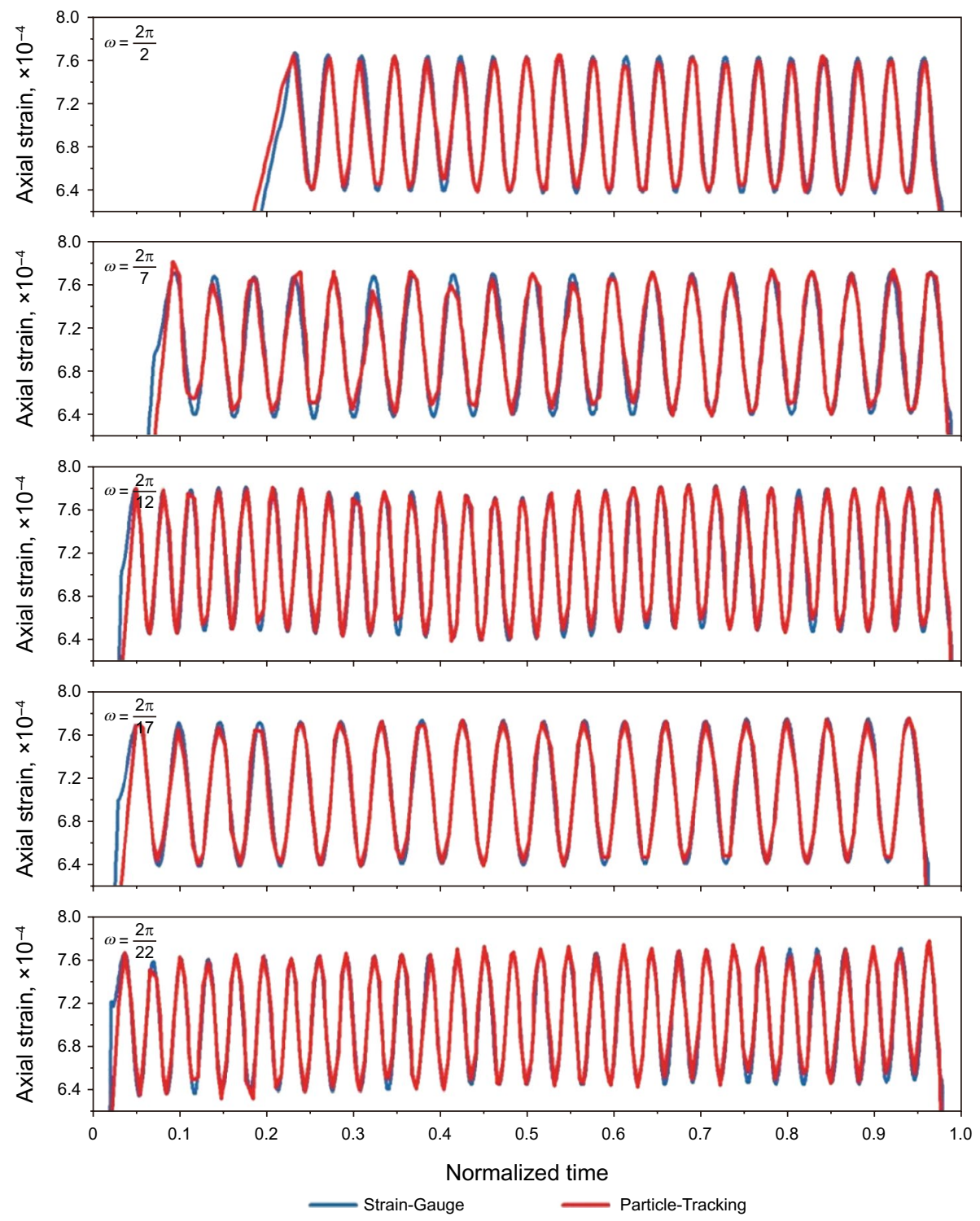

Fig. 9 Comparison of axial strains measured by strain gauges and particle-tracking

the mechanical behavior of flexible pipes is feasible although frictional effect is not considered.

To evaluate how experimental outcomes differ from the model, the maximum and minimum values of the axial strain, as well as the amplitude, given by measurements from strain gauges and theoretical model, respectively, are compared as shown in Fig. 12.

The model results show that the larger the value of frequency, the smaller the amplitude is, whereas no clear relation can be found in experiment. It should be noted that, because the peak and valley values of axial strain in each cycle are obviously different in experiment, this comparison requires the use of mean values rather than one group, which are slightly larger than the ones provided by the model. One of the reasons for this little difference may be caused by the extrusion of the polymeric element into the near helical steel wires, which is equivalent to increase the axial load on the sample. Moreover, there is no significant difference in amplitude between the experiment and theoretical model for each case. All those satisfactory agreements indicate that the model proposed in this paper is coherent.

\section{Conclusions}

Mechanical experiments for evaluating the axial structural damping behavior of flexible pipes have been performed. Results demonstrate the existence of hysteresis behavior when an axial oscillation force is exerted on the sample. Loss factor representing structural damping is obtained 


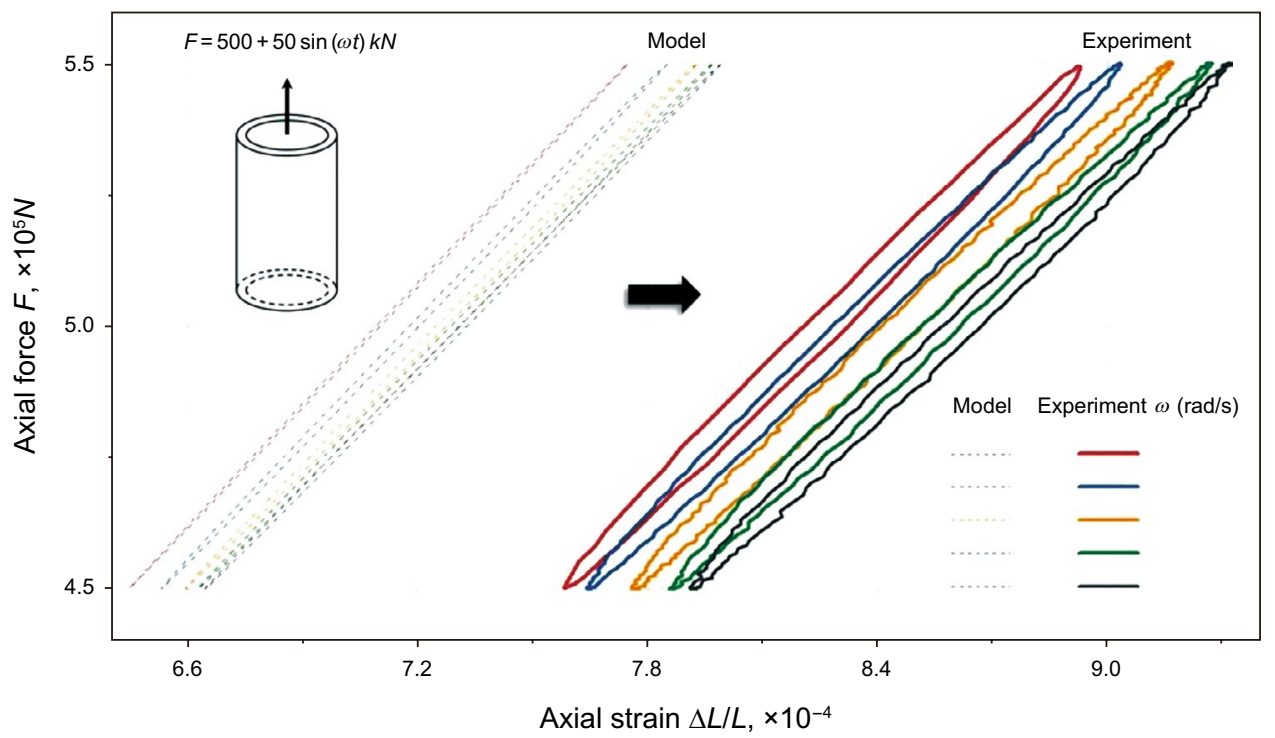

Fig. 10 Comparison of axial force-strain relations generated by LVDT results and theoretical model given by Liu and Vaz (2016b)

Table 2 Results of loss factor and equivalent axial stiffness given by experiment and model

\begin{tabular}{|c|c|c|c|c|c|c|c|c|c|c|c|c|}
\hline \multirow[t]{2}{*}{$F 077, \mathrm{rad} / \mathrm{s}$} & \multicolumn{2}{|c|}{ Min. strain $\left(\times 10^{-4}\right)$} & \multicolumn{2}{|c|}{ Max. strain $\left(\times 10^{-4}\right)$} & \multicolumn{2}{|c|}{$\begin{array}{l}\text { Potential energy, } \\
\mathrm{Nm}\end{array}$} & \multicolumn{2}{|c|}{$\begin{array}{l}\text { Dissipated energy, } \\
\mathrm{Nm}\end{array}$} & \multicolumn{2}{|c|}{ Loss factor, $\%$} & \multicolumn{2}{|c|}{$\begin{array}{l}\text { Equivalent axial } \\
\text { stiffness }\left(\times 10^{8} \mathrm{~N}\right)\end{array}$} \\
\hline & Exp. & Model & Exp. & Model & Exp. & Model & Exp. & Model & Exp. & Model & Exp. & Model \\
\hline $2 \pi / 2$ & 7.58 & 6.45 & 8.93 & 7.75 & 1.69 & 1.62 & 0.88 & 0.10 & 8.31 & 0.94 & 7.41 & 7.70 \\
\hline $2 \pi / 7$ & 7.64 & 6.53 & 9.04 & 7.85 & 1.74 & 1.65 & 0.86 & 0.21 & 7.88 & 2.06 & 7.18 & 7.59 \\
\hline $2 \pi / 12$ & 7.76 & 6.60 & 9.17 & 7.93 & 1.77 & 1.67 & 0.83 & 0.21 & 7.46 & 2.01 & 7.07 & 7.51 \\
\hline $2 \pi / 17$ & 7.86 & 6.63 & 9.27 & 7.97 & 1.77 & 1.68 & 0.81 & 0.19 & 7.32 & 1.76 & 7.07 & 7.46 \\
\hline $2 \pi / 22$ & 7.91 & 6.65 & 9.32 & 7.99 & 1.76 & 1.68 & 0.79 & 0.16 & 7.16 & 1.53 & 7.10 & 7.44 \\
\hline
\end{tabular}

(a)

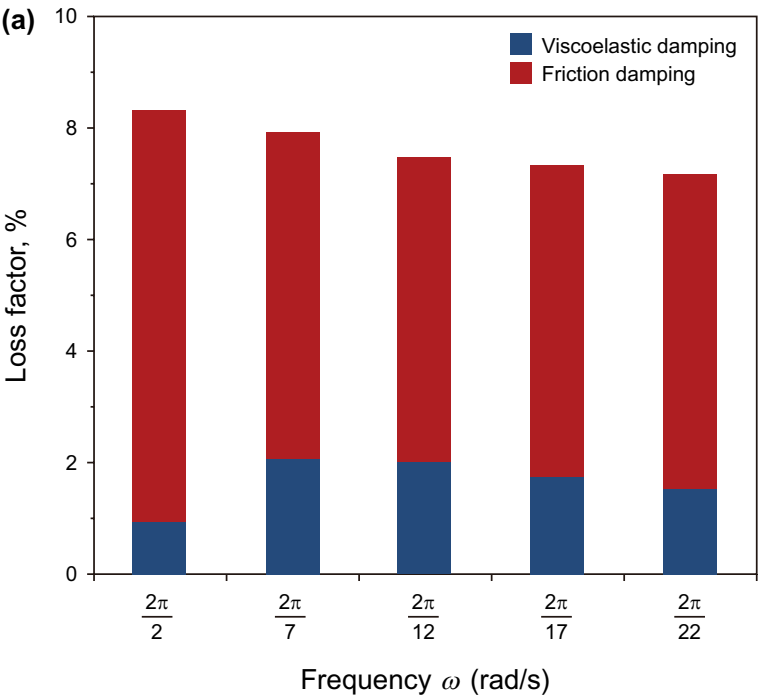

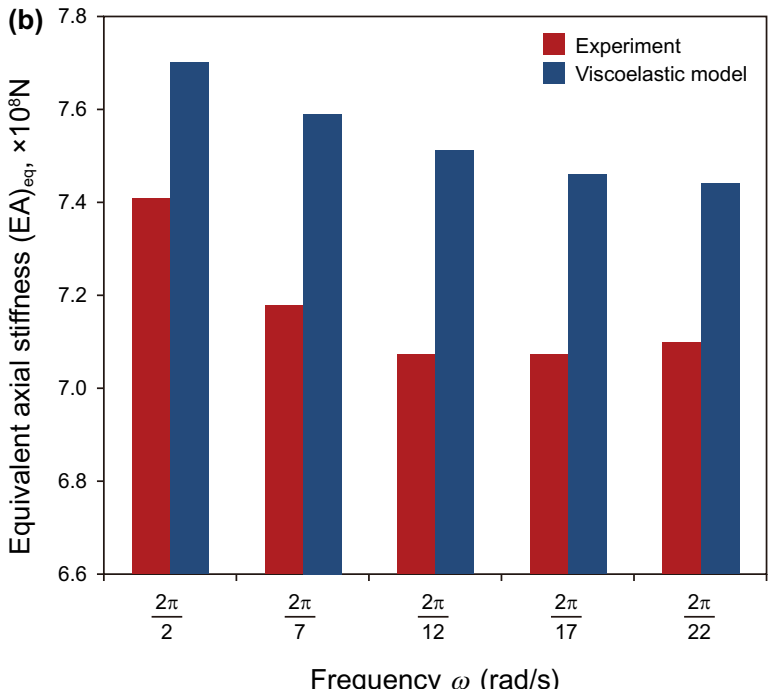

Fig. 11 Comparison of axial structural damping behaviors given by experimental results and theoretical model (a loss factor; b equivalent axial stiffness) 

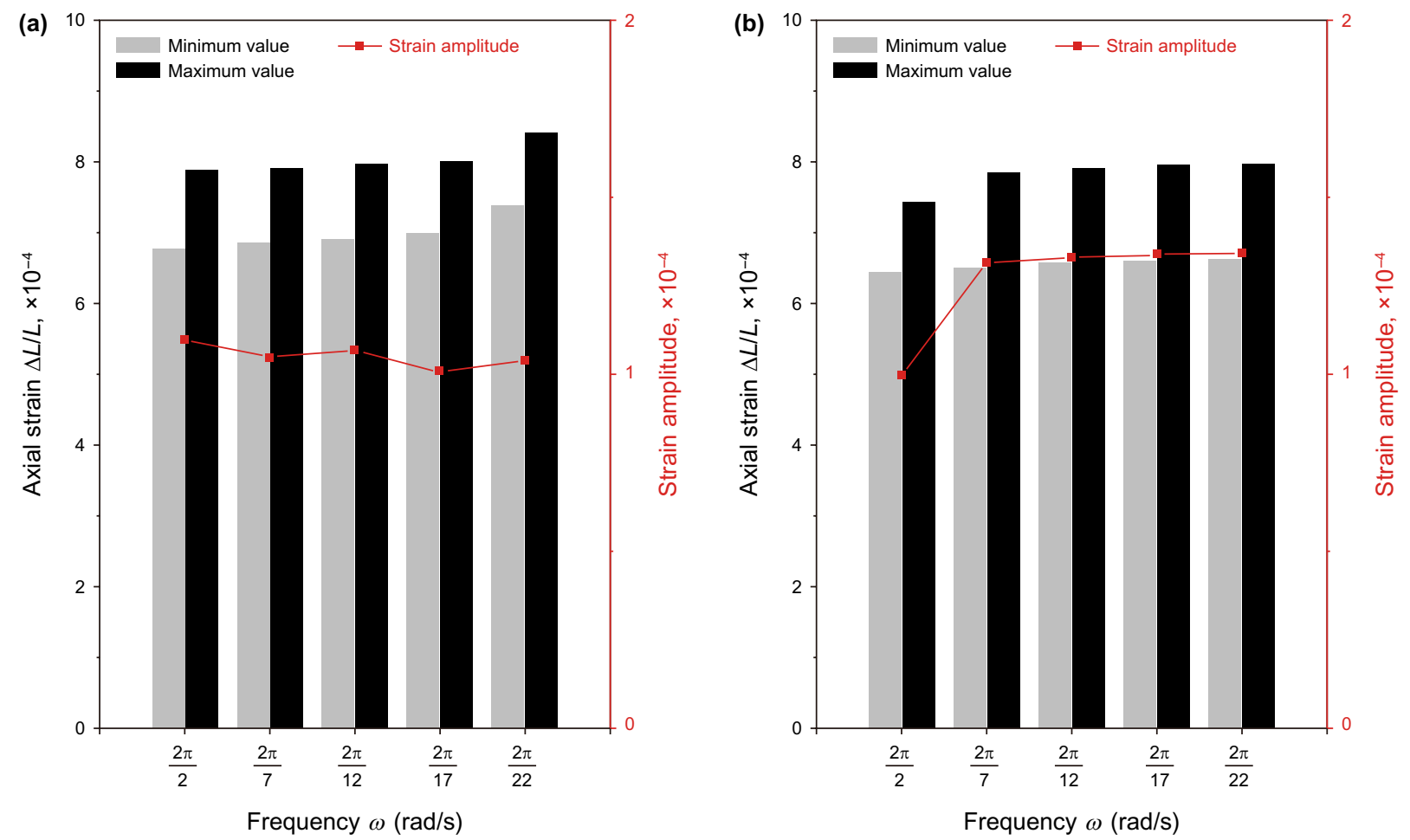

Fig. 12 The minimum and maximum values of axial strain, and their difference (a test results; $\mathbf{b}$ theoretical results)

through force-elongation plot, which is considered as a sum of frictional and viscoelastic damping. It is seen that the value of loss factor decreases as the loading frequency declines, but maybe tend to some stable value of $7 \%$. The viscoelastic axisymmetric model, in the form of a linear system of equations, has provided an effective way for capturing the value of loss factor representing the viscoelastic damping. Finding shows, compared with friction effect, the contribution of the polymeric components in flexible pipe to the structural damping behavior is relatively small. The proportion of viscoelastic damping is less than $25 \%$ of total damping. It indicates that internal friction mechanisms presumably exert a large influence on the measurement of loss factor, representing around $75 \%$ of the total damping. Moreover, the results presented by both model and strain gauges denote that reduced values of frequency relative to tensile load lead to elevated amplitude of axial strains and thus to a decrease in equivalent axial stiffness. This demonstrates that the proposed approach can represent an excellent method of viscoelastic analysis for flexible pipes. It should also be stressed that axial damping should be included in the qualification program of a flexible pipe.

Acknowledgements The authors acknowledge the support from the National Natural Science Foundation of China (Youth Program) (Grant No. 51809276), and the National Key Research and Development
Plan of China (Grant No. 2018YFC0310504) and CNPq - National Council of Scientific and Technological Development (Grant No. 302380/2013-2).

Open Access This article is licensed under a Creative Commons Attribution 4.0 International License, which permits use, sharing, adaptation, distribution and reproduction in any medium or format, as long as you give appropriate credit to the original author(s) and the source, provide a link to the Creative Commons licence, and indicate if changes were made. The images or other third party material in this article are included in the article's Creative Commons licence, unless indicated otherwise in a credit line to the material. If material is not included in the article's Creative Commons licence and your intended use is not permitted by statutory regulation or exceeds the permitted use, you will need to obtain permission directly from the copyright holder. To view a copy of this licence, visit http://creativecommons.org/licenses/by/4.0/.

\section{References}

Chenouard N, Smal I, De Chaumont F, et al. Objective comparison of particle tracking methods. Nat Methods. 2014;11(3):281. https:// doi.org/10.1038/nmeth.2808.

Custódio AB, Vaz MA. A nonlinear formulation for the axisymmetric response of umbilical cables and flexible pipes. Appl Ocean Res. 2002;24(1):21-9. https://doi.org/10.1016/S0141-1187(02)00007 $-\mathrm{X}$.

Ebrahimi A, Kenny S, Hussein A. Finite element investigation on the tensile armor wire response of flexible pipe for axisymmetric loading conditions using an implicit solver. J Offshore Mech Arct Eng. 2018;140(4):041402. 
Feret JJ, Bournazel CL. Calculation of stresses and slip in structural layers of unbonded flexible pipes. J Offshore Mech Arct Eng. 1987;109(3):263-9.

Guedes RM. Nonlinear viscoelastic analysis of thick-walled cylindrical composite pipes. Int J Mech Sci. 2010;52(8):1064-73. https://doi. org/10.1016/j.ijmecsci.2010.04.003.

Kebadze E. Theoretical modelling of unbonded flexible pipe crosssections. South Bank University; 2000.

Kumar CK, Agarwal A, Chillarige RR. Color and texture image segmentation. In: International workshop on multi-disciplinary trends in artificial intelligence. Berlin, Heidelberg: Springer; 2012. p. 69-80.

Liang X, Zha X, Jiang X, et al. Semi-analytical solution for dynamic behavior of a fluid-conveying pipe with different boundary conditions. Ocean Eng. 2018;163(SEP.1):183-90. https://doi. org/10.1016/j.oceaneng.2018.05.060.

Liang X, Zha X, Yu Y, et al. Semi-analytical vibration analysis of FGM cylindrical shells surrounded by elastic foundations in a thermal environment. Compos Struct. 2019;223(SEP.):110997.1-110997.11.

Liu J, Vaz MA. Axisymmetric viscoelastic response of flexible pipes in time domain. Appl Ocean Res. 2016a;55:181-9. https://doi. org/10.1016/j.apor.2015.12.003.

Liu J, Vaz MA. Viscoelastic axisymmetric structural analysis of flexible pipes in frequency domain considering temperature effect. Marine Struct. 2016b;50:111-26. https://doi.org/10.1016/j.marst ruc.2016.07.003.

Pérez P, Hue C, Vermaak J, et al. Color-based probabilistic tracking. In: European conference on computer vision. Berlin, Heidelberg: Springer; 2002. p. 661-75.

Pesce CP, Ramos Jr R, Yamada da Silveira LM, et al. Structural behavior of umbilicals - part I: mathematical modeling. OMAE2010$20892 ; 2010$
Ramos R, Kawano A. Local structural analysis of flexible pipes subjected to traction, torsion and pressure loads. Marine Struct. 2015;42:95-114. https://doi.org/10.1016/j.marstruc.2015.03.004.

Ramos R, Martins CA, Pesce CP, et al. Some further studies on the axial-torsional behavior of flexible risers. J Offshore Mech Arct Eng. 2014;136(1):011701.

Sævik S. Theoretical and experimental studies of stresses in flexible pipes. Comput Struct. 2011;89(23):2273-91. https://doi. org/10.1016/j.marstruc.2012.03.005.

Santos CCP, Pesce CP, Salles R, et al. An experimental assessment of the hysteresis behavior of umbilical cables under cyclic traction. ASME. 2017;2017:V05AT04A032. https://doi.org/10.1115/ OMAE2017-62081.

Schneider CA, Rasband WS, Eliceiri KW. NIH image to ImageJ: 25 years of image analysis. Nat Methods. 2012;9(7):671-5.

Silveira LMY, Tanaka RL, Novaes JPZ. Rayleigh damping effects on global analysis of umbilicals. In: 30th international conference on ocean, offshore and Arctic engineering. American Society of Mechanical Engineers; 2011. p. 549-59. https://doi.org/10.1115/ omae2011-49584.

Ungar EE, Kerwin EM Jr. Loss factors of viscoelastic systems in terms of energy concepts. J Acoust Soc Am. 1962;34(7):954-7.

Witz JA, Tan Z. On the axial-torsional structural behaviour of flexible pipes, umbilicals and marine cables. Marine Struct. 1992;5(23):205-27. https://doi.org/10.1016/0951-8339(92)90029-O.

Ye W, Wang C, Yan FA. Color-based target tracking hybrid algorithm by combining Kalman filter and particle filter. J Comput Inf Syst. 2013;9(5):1743-50. 